\title{
SEMIQUANTITATIVE ASSESSMENT OF DISHEVELLED-3 PHOSPHORY- LATION STATUS BY MASS SPECTROMETRY
}

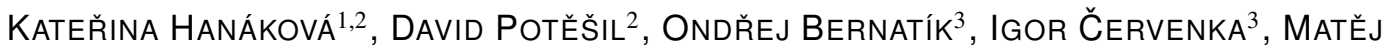 \\ RÁDSETOULAL ${ }^{3}$, VÍTĚZSLAV BRYJA ${ }^{3}$, AND ZBYNĚK ZDRÁHAL *1,2 \\ ${ }^{1}$ National Centre for Biomolecular Research, Faculty of Science, Masaryk University, Brno, 62500, CZECH \\ REPUBLIC \\ ${ }^{2}$ Central European Institute of Technology, Masaryk University, Brno, 62500, CZECH REPUBLIC \\ ${ }^{3}$ Department of Experimental Biology, Faculty of Science, Masaryk University, Brno, 62500, CZECH \\ REPUBLIC
}

\begin{abstract}
The focus of this paper is the human Dishevelled 3 protein ( $h D v l 3$ ), an essential component of the Wnt signalling pathway that contributes to their regulation. Mass spectrometry-based analysis of hDvl3 phosphorylations induced by eight associated kinases was performed revealing several dozens of phosphorylation sites. The main outcome of this study was the description of Dvl phosphorylation "patterns" induced by individual kinases.
\end{abstract}

Keywords: phosphorylation, Dishevelled 3, mass spectrometry, CK1 $\varepsilon, \mathrm{NEK} 2$

\section{Introduction}

Reversible protein phosphorylation belongs to posttranslational modification which is of high biological significance mainly due to its role in regulating cellular processes such as gene expression, cell division, signal transduction, metabolism, differentiation, apoptosis, etc. [1]

Dishevelled-3 (Dvl3) is a protein involved in the Wnt signalling pathways and gets heavily phosphorylated in response to pathway activation by Wnt ligands [2].

In our study, sodium dodecyl sulfate polyacrylamide gel electrophoresis (SDS-PAGE) separation of hDvl3 immunoprecipitates was combined with titanium dioxide phosphopeptide enrichment followed by liquid chromatography-tandem mass spectrometry (LCMS/MS). Data were processed using Skyline software to obtain a semiquantitative assessment with regard to the occupancy of phosphorylation sites induced by individual kinases.

\section{Experimental}

\subsection{Cell culture and transfection}

The cell line HEK293 was used for this study. Cells were seeded in dishes $15 \mathrm{~cm}$ in diameter and transfected at a confluence of $60 \%$. Two days after transfection, after reaching confluence, they were harvested for immunoprecipitation. A chilled NP-40 lysis buffer with protease inhibitors, dithiothreitol (DTT), phosphatase inhibitors and

\footnotetext{
*Correspondence: zdrahal@sci.muni.cz
}

N-ethylmaleimide (NEM) was used for cell lysis. The lysate was incubated with the antibody Anti FLAG M2.

\subsection{Gel electrophoresis, protein digestion and phosphopeptide enrichment}

Immunoprecipitates were separated by SDS-PAGE, fixed with acetic acid in methanol, and stained with Coomassie Brilliant Blue for 1 hour then partially destained.

Corresponding 1-D bands were excised. After destaining, the proteins in gel pieces were incubated with $10 \mathrm{mM}$ of DTT at $56{ }^{\circ} \mathrm{C}$ for 45 mins. After the removal of DTT excess samples were incubated with $55 \mathrm{mM}$ of iodoacetamide (IAA) at room temperature in darkness for 30 mins, then an alkylation solution was removed and gel pieces were hydrated for $45 \mathrm{mins}$ at $4{ }^{\circ} \mathrm{C}$ in a digestion solution $(5 \mathrm{ng} / \mu \mathrm{l}$ trypsin, sequencing grade, Promega, Fitchburg, Wisconsin, USA, in $25 \mathrm{mM}$ ammonium bicarbonate $(\mathrm{AmBic}))$. The trypsin digestion proceeded for 2 hours at $37^{\circ} \mathrm{C}$ on a Thermomixer (at $750 \mathrm{rpm}$; Eppendorf AG, Hamburg, Germany). Subsequently, the tryptic digests were cleaved by chymotrypsin $(5 \mathrm{ng} / \mu \mathrm{l}$, sequencing grade, Roche, Basel, Switzerland, in $25 \mathrm{mM}$ AmBic) for 2 hours at $37{ }^{\circ} \mathrm{C}$. Digested peptides were extracted from gels using 50\% acetonitrile (ACN) solution with $2.5 \%$ formic acid (FA) and concentrated in a SpeedVac concentrator (Eppendorf AG, Hamburg, Germany). The aliquot (1/10) of the sample was transferred to an autosampler vial and concentrated under a vacuum. Water was used to produce $15 \mu \mathrm{l}$ of the peptide solution which was directly 


$\begin{array}{ll}\text { MGETKIIYHLDGQETPYLVKLPLPAERVTLADFKGVLQRPSYKFFFKSMD } & 50 \\ \text { DDFGVVKEEISDDNAKLPCFNGRVVSWLVSAEGSHPDPAPFCADNPSELP } & 100 \\ \text { PPMERTGGIGDSRPPSFHPHAGGGSQENLDNDTETDSLVSAQRERPRRRD } & 150 \\ \text { GPEHATRLNGTAKGERRREPGGYDSSSTLMSELETTFFDSDEDDSTSR } & 200 \\ \text { FSSSTEQSSASRLMRRHKRRRRKQKVSRIERSSSFSSITDSTMSLNIITV } & 250 \\ \text { TLNMEKYNFLGISIVGQSNERGDGGIYSIMKGGAVTADGRIEPGDMLL } & 300 \\ \text { QVNEINFENMSNDDAVRVLREIVHKPGPITLTVAKCWDPSPRGCFTLPRS } & 350 \\ \text { EPIRPIDPAAWVSHTAAMTGTPAYGMSPLSTITSTSSITSSIPDTER } & 400 \\ \text { LDDFHLSIHSDMAAIVKAMASPESGLEVRDRMWLKITIPNAFIGSDVVDW } & 450 \\ \text { LYHNVEGFTDRREARKYASNLLKAGFIRHTVNKITFSEQCYYIFGDLCGN } & 500 \\ \text { MANLSLHDHDGSSGASDQTLAPLPHPGAAPWPMAFPYQYPPPPHPYNPH } & 550 \\ \text { PGFPELGYSYGGGSASSQHSEGSRSSGSNRSGSDRRKEKDPKAGDSKSGG } & 600 \\ \text { SGSESDHTTRSSLRGPRERAPSERSGPAASEHSHRSHHSLASSLRSHHTH } & 650 \\ \text { PSYGPPGVPPLYGPPMLMMPPPPAAMGPPGAPPGRDLASVPPELTASRQS } & 700 \\ \text { FRMAMGNPSEFFVDVM } & \end{array}$

Figure 1: Comparison of phosphorylation sites of hDv13 induced by $\mathrm{CK} 1 \varepsilon$ and NEK2. Experimentally determined phosphorylation sites by $\mathrm{CK} 1 \varepsilon$ and NEK2 are indicated by green background color. Phosphorylation sites identified only by NEK2 are indicated by red background color.

analyzed by liquid chromatography coupled with mass spectrometry (LC-MS/MS) for protein identification (ID run).

The rest of the peptide mixture was used for phosphopeptide analysis. MS PhosphoMix 1, 2, 3 Light (Sigma Aldrich) was added to the samples before the phosphopeptide enrichment step in a concentration of 0.1 pmol. Phosphopeptides were enriched using a Pierce Magnetic Titanium Dioxide Phosphopeptide Enrichment Kit (Thermo Fisher Scientific, Waltham, Massachusetts, USA) according to the protocol of the manufacturer and eluted into an autosampler vial. The solution was concentrated under a vacuum to a volume of less than $5 \mu \mathrm{l}$, dissolved in water and $0.6 \mu \mathrm{l}$ of $5 \%$ FA was used to obtain $15 \mu \mathrm{l}$ of the peptide solution before LC-MS/MS analysis.

\subsection{Mass spectrometry}

LC-MS/MS analyses of the peptide mixture were conducted using a RSLCnano system connected to a Orbitrap Elite hybrid mass spectrometer (Thermo Fisher Scientific) with a ABIRD (Active Background Ion Reduction Device; ESI Source Solutions) and a Digital PicoView DPV550 (New Objective) ion source (tip rinsing by $50 \%$ ACN with $0.1 \%$ FA) installed. Prior to LC separation, tryptic digests were concentrated online and desalted using a trapping column $(100 \mu \mathrm{m} \times 30 \mathrm{~mm})$ filled with 3.5- $\mu$ m of XBridge BEH $130 \AA$ C18 sorbent (Waters). After the trapping column was washed with $0.1 \% \mathrm{FA}$, the peptides were eluted (flow rate of $300 \mathrm{nl} / \mathrm{min}$ ) from the trapping column onto a Acclaim PepMap100 C18 column $(3 \mu \mathrm{m}$ particles, $75 \mu \mathrm{m} \times 500 \mathrm{~mm}$; Thermo Fisher Scientific) along a 65 min-long gradient. Mobile phase A $(0.1 \%$ FA in water $)$ and mobile phase $\mathrm{B}(0.1 \% \mathrm{FA}$ in $80 \% \mathrm{ACN}$ ) were used in both cases. The gradient elution started at $1 \%$ of mobile phase B and increased from $1 \%$ to $56 \%$ during the first 50 mins $(30 \%$ in the 35 th and $56 \%$ in the 50 th $\mathrm{min}$ ), then increased linearly to $80 \%$ of mobile phase B over the following 5 mins and remained at this state for the next 10 mins. Equilibration of the trapping column and the anlytical column was conducted prior to injection of the sample into the sample loop. The outlet of the analytical column was directly connected to the Digital PicoView DPV550 ion source.

MS data were acquired in a data-dependent strategy by selecting the top 6 precursors based on precursor abundance in the survey scan $(350-2000 \mathrm{~m} / \mathrm{z})$. The resolution of the survey scan was 60,000 (at $400 \mathrm{~m} / \mathrm{z}$ ) with a target value of $1 \times 10^{6}$ ions, one microscan and a maximum injection time of $200 \mathrm{~ms}$. High resolution (resolution of 15,000 at $400 \mathrm{~m} / \mathrm{z}$ ) higher energy collisional dissociation (HCD) MS/MS spectra were acquired with a target value of 50,000. The normalized collision energy was $32 \%$ for HCD spectra. The maximum injection time for MS/MS was $500 \mathrm{~ms}$. Dynamic exclusion was enabled for $45 \mathrm{~s}$ after the acquisition of one MS/MS spectra and early expiration was disabled. The isolation window for MS/MS fragmentation was set to $2 \mathrm{~m} / \mathrm{z}$.

The analysis of the mass spectrometric RAW data files was carried out using the Proteome Discoverer software (Thermo Fisher Scientific; version 1.4) with utilization of the in-house Mascot (Matrixscience; version 2.4.1) search engine. MS/MS ion searches were conducted against an in-house database containing the expected protein of interest with additional sequences from the cRAP (common Repository of Adventitious Proteins) database (downloaded from http://www.thegpm.org/crap/). Mass tolerance for peptides and MS/MS fragments were $7 \mathrm{ppm}$ and 0.03 Da, respectively. Oxidation of methionine, deamidation $(\mathrm{N}, \mathrm{Q})$ 

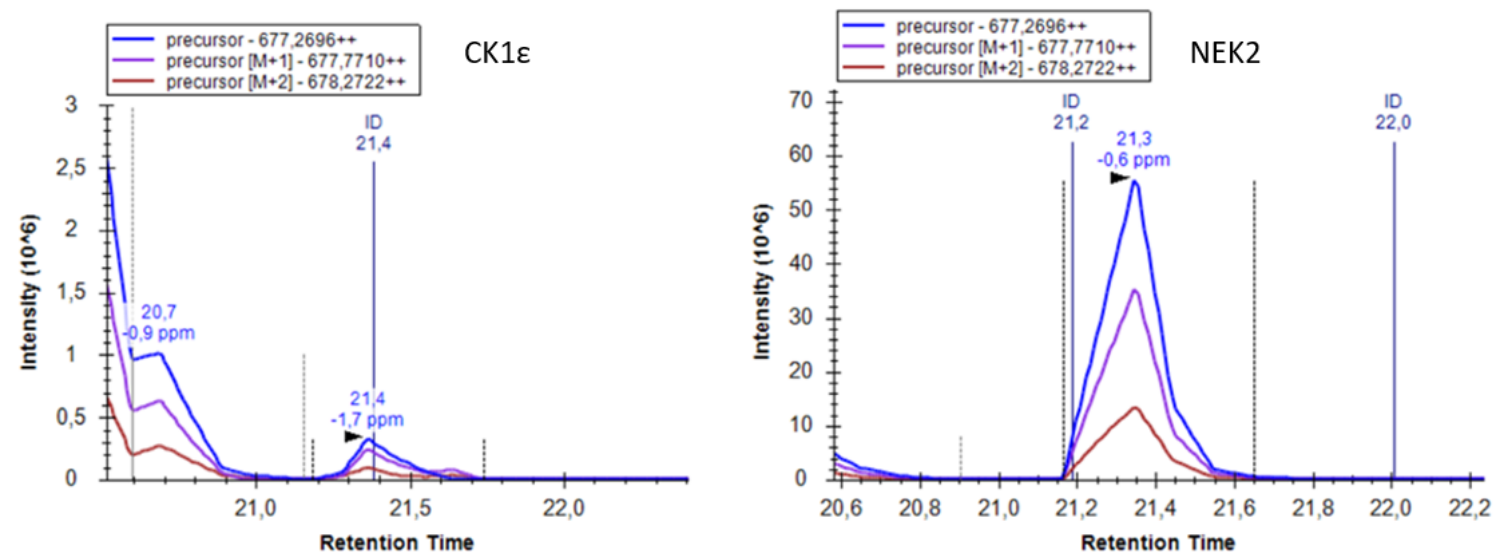

Figure 2: Semiquantitative analysis of phosphorylation site serine 204 (S204) on peptide FSSpSTEQSSASR induced by CK1 $\varepsilon$ and NEK2. Precursor and selected fragment traces of corresponding hDvl3 phosphopeptides are shown for CK1 $\varepsilon$ and NEK2 (in Skyline). The highest signal intensity was detected in the case of NEK2.

and phosphorylation (S, T, Y) as optional modifications, carbamidomethylation of $\mathrm{C}$ as a fixed modification and three miss cleavages of enzymes were set for all searches. The phosphoRS feature was used for phosphorylation site localization.

Quantitative information was assessed and manually validated in Skyline software (Skyline-daily 3.1.1.8884).

\section{Results and Analysis}

\subsection{Identification of phosphorylation sites}

Phosphorylation is important for protein function and regulation. The phosphorylation status of human Dvl3 induced by eight individual Ser/Thr kinases that were previously reported or identified by an unbiased MS screen for Dvl-associated kinases was analysed. Dvl3 contains 131 serines/threonines, which can be potentially phosphorylated. In total, $88 \mathrm{Ser} / \mathrm{Thr}$ phosphorylation sites and one tyrosine phosphorylation site in Dvl3 were identified.

\subsection{Phosphorylations induced by $C K 1 \varepsilon$ and NEK2}

Based on our experiment, a phosphorylation "map" of the Dvl protein was created that described the complex phosphorylation "fingerprint" for each kinase tested. Eight of the kinases used to induce phosphorylation include CK1 $\varepsilon$ and NEK2. Fig. 1 shows a qualitative comparison of the identified phosphorylation sites using these two kinases. In the case of $\mathrm{CK} 1 \varepsilon$ induction, 77 phosphorylation sites were identified, and in the case of NEK2, 87 phosphorylation sites were determined from a total of 131 possible Ser/Thr phosphorylation sites in the Dvl3 protein.

Next in terms of qualitative characterization, a semiquantitative comparison with regard to the occupancy of phosphorylation sites induced by individual kinases was conducted. The Skyline software was used for this evaluation. The individual phosphorylated peptides were compared based on their peak areas. A comparison of a selected peptide phosphorylated in the position of S204 by $\mathrm{CK} 1 \varepsilon$ and NEK2 is shown in Fig. 2. The peak area was determined for CK1 $\varepsilon$ as 7.60e6 and for NEK2 as 1.07e9. Subsequently, double normalization of the data was performed using a set of phosphopeptide standards (added to the sample prior to the phospho-enrichment step) and by unphosphorylated peptides identified in the identification run. The resulting areas (CK1 $\varepsilon$ : $1.23 \mathrm{e} 7$ and NEK2: $1.10 \mathrm{e} 9)$ were compared with each other.

\section{Discussion}

Our study focused on the determination of the phosphorylation sites of Dvl3 by MS induced by eight kinases. $88 \mathrm{Ser} / \mathrm{Thr}$ phosphorylations from a total of 131 sites and 1 tyrosine phosphorylation were identified which can be potentially phosphorylated.

$\mathrm{CK} 1 \varepsilon$-induced phosphorylation was identified at 77 unique sites and 10 more phosphorylation sites were induced by NEK2. Previous studies in various experimental systems identified several phosphorylation sites spread throughout the structure of the protein Dvl3 [3,4]. Our data clearly demonstrate that the phosphorylation of the protein Dvl3 is extensive and the number of phosphorylated sites exceeds 60 .

\section{Conclusion}

An approach based on the SDS-PAGE separation of Dvl3 immunoprecipitates, $\mathrm{TiO}_{2}$ phospho-enrichment followed by LC-MS/MS analysis and data processing using Skyline software was utilized for the evaluation of semiquantitative differences in the phosphorylation level of $\mathrm{hDvl} 3$ at particular sites within the set of eight selected kinases. 
Differences were observed in terms of the phosphorylation profiles induced by individual kinases, as indicated in Fig. 1. Based on our results, a "comprehensive map" of the phosphorylations of human Dvl3 will be created.

\section{Acknowledgement}

This work was carried out with the support of the project CEITEC 2020 (LQ1601) funded by the Ministry of Education, Youth and Sports (MEYS) of the Czech Republic under the National Sustainability Programme II. The Czech Infrastructurefor Integrative Structural Biology (CIISB) research infrastructure project LM2015043 funded by MEYS is gratefully acknowledged for financially supporting our LC-MS/MS measurements at the Proteomics Core Facility. The support from the Czech Science Foundation project no. 15-21789S is also gratefully acknowledged.

\section{REFERENCES}

[1] Kersten, B., Agrawal, G. K., Iwahashi, H., Rakwal, R.: Plant phosphoproteomics: A long road ahead, Proteomics, 2006 6(20), 5517-5528 DOI: 10.1002/pmic.200600232

[2] Bernatík, O., Šedová, K., Schille, C., Ganji, S. R., Červenka, I., Trantírek, L., Schambony, A., Zdráhal, Z., Bryja, V.: Functional analysis of Dishevelled3 phosphorylation identifies distinct mechanisms driven by casein kinase 1 epsilon and frizzled5, J. Biol. Chem., 2014 34(289), 23520-23533 DOI: 10.1074/jbc.M114.590638

[3] Yanfeng, W. A., Berhane, H., Mola, M., Singh, J., Jenny, A., Mlodzik, M.: Functional dissection of phosphorylation of Disheveled in Drosophila, Dev. Biol., 2011 360, 132-142 DOI: 10.1016/j.ydbio.2011.09.017

[4] Klimowski, L. K., Garcia, B. A., Shabanowitz, J., Hunt, D. F., Virshup, D. M.: Site-specific casein kinase $1 \epsilon$-dependent phosphorylation of Dishevelled modulates $\beta$-catenin signaling, FEBS J., 2006 273, 4594-4602 DOI: 10.1111/j.1742-4658.2006.05462.x 